\title{
The Macroscopic Analysis of Language Perception*
}

\author{
Jiangping Zhou \\ China West Normal University \\ Nanchong, China
}

\begin{abstract}
Language perception is the key step to psychological process in language use and the important topic in psycho-linguistic study; and it is also the major way for human beings to master and understand language. This paper analyzed language perception from the three aspects of definitions, psychological features and schemes of language perception.
\end{abstract}

Keywords-macro-scope; psycholinguistics; language
perception

\section{INTRODUCTION}

Psycholinguistics is a subject that integrally applies linguistic and psychological theories and experimental methods, and supervises the psychological mechanism and psychological process of language acquisition, learning and use. The participants of language perception are actually "speech". Language is the social product of language mechanism and the subjective phenomenon of social life. As to specific language users, it is a unified, shared and necessary regulation that every language holds for pronunciation, grammar and syntax. The moment the regulation is formed, it is of great stability. However, speech is a psychological phenomenon with individuality and diversification, which demonstrates the psychological communication process. This paper intends to analyze language perception from the three aspects of definitions, psychological features and schemes of language perception.

\section{Definition OF LANGUAGE PERCEPTION}

Language perception is a very important concept, which focuses on speech perception, understanding and beholding, and it is concerned with language development and speech production and understanding (Wang, 2000). Humboldt (1997) held that the key to language form was to make meaning mix with sound, to make auditory sense be retrieved from sound completely, and thus abandoned all these irrelevant to meaning and confined sound solely according to meaning. He believed that, during the process of speech perception, meaning was integrally retrieved from sound. Sapir (1985) also believed that language was mainly an audio-semiotic system. Its auditory sense and perception in speech was what we were talking about language perception. He also held that auditory sense had a function of "translation" to its relevant sounds, and therefore, gained the

*This paper is funded by the Federation of Social Sciences, Nanchong China (Project No., NC2012C013). perception from the speech.

Besides that the language perception was analyzed from the aspect of auditory sense, some other scholars illustrated language perception from sense. They defined language perception as "words' sensory feelings", that is, when the word "green" appears, not only will we think of the color, but also we will feel numerous interesting meanings as "hopes, natural contributions and juvenile spirit", and so on (Ye,1980). Zhu (1996), holding the similar point of views, also believed that language perception was the sensitivity to language words.

Some studies define language perception from subjective intuition. For instance, Lu (2006) believed that language perception was a process of complex cognitive and aesthetic activity initiated from language words, an intuitive ability to master language words, and a combination of sensibility and rationality. Pan (1995) proposed that language perception is a process of complex psychological and epistemic activity caused by language words, including such psychological factors as feeling, intuition, memory and thinking. Hu (1992) exemplified that language perception was a synthesis of intuitive constituents, fixed constituents and dynamic constituents, and it was also an instrumental mechanism that formed from speech practice and again assigned to supervise and regulate speech practice.

The above mentioned studies refined language perception from the three aspects of auditory sense, feeling and intuition, which provided us with perspectives of understanding language perception from different facets.

\section{The PsychologicAl FEATURES OF LANGUAGE PERCEPTION}

What psychological features does language perception have? From the perspective of language teaching, as a dynamic behavioral process, the features of language perception is transference; as a static structural state, its features are schemes (Li, 2000). Specifically, everyone has his or her unique "individual dictionary", which records their own lexicons and grammatical systems. Structurally, it is a network structure.

Just as Ye (1980) said that when the word "green" appeared, not only would we think of the color, but also we would feel numerous interesting meanings as "hopes, natural contributions and juvenile spirit", and so on. It is actually a psychological transference. Furthermore, another feature of language perception is scheme, which is a psychological 
structure that the subject exists in advance in speech activity in the form of speech information ( $\mathrm{Li}, 2000)$.

Additionally, Pan (1995) proposed that the psychological structure of language perception possess the features of sensitivity, implicitness and vagueness. It is sensitive because that language perception can perceive speech information stimuli suddenly; it is implicit because that language perception can only perceive these features by decoding linguistic signs; it is vague because that language itself is uncertain, changing and random.

\section{SCHEMES OF LANGUAGE PERCEPTION}

Schemes of language perception are constructed on the basis of schematic theories and they are playing an important role in the process of speech perception, understanding and gaining.

\section{A. Li's Scheme of Language Perception}

Li (2000) believed that the psychological mechanism of language was the scheme of language perception, which was subsequently constructed among speech users and was regular, open and organized. However, its regularity appears in a specific phase temporarily. Generally speaking, it is a circular process from regularity and stability to irregularity and instability, and then back to regularity and stability again. The most obvious feature of schemes of language perception is openness, because the schemes are exchanging information continuously with outside world. The appearance of new information will break the temporary balance of the schemes, and thus the contradictions in schemes are stimulated. Certainly, the contradictions will give rise to the reorganization among these factors in the schemes, which is of automation for schemes to gain new information so as to keep balance among these constituents. The scheme is organized because its relevant constituents will locate the new information into proper position, and automatically associate themselves with the matched original constituents. New information is put in by two ways of clockwise and counterclockwise. The former supplements and consolidates the scheme of language perception, while the latter is rejected by scheme. Under the circumstance, the subject achieves the input by ways of either obeying to speech users, or adjusting the scheme of language perception, or reconstructing (2000).

\section{B. Wang's Scheme of Language Perception}

Wang proposed that the scheme of language perception was the basement for language perception to be produced, and it was a huge, delicate and intricate network system, including many interactive "knowledge" units (2000). The scheme is composed of three levels of structural networks. Level one: form structure; level two: situation structure; level three: intension structure.

Speech form, in contrast with level one, is composed of two systems: sound form and meaning form. The former is relating to the scheme of phoneme, prosody, stress and intonation. Each individual possesses his or her unique and relevant scheme of phoneme with the language he or she uses. If the sound of speech users belongs to the scope of a specific phoneme and the reader or the listener shares the similar scheme of phoneme, then the reader or the listener can retrieve it and confirm its meaning successfully. The recognition of sound, certainly, needs some other factors' collaboration, for instance, syntax and word meaning. The sound is not secluded, but successive. When the phoneme is divergent or even missing, the reader or the listener, with the help of syntax and word meaning, can perceive speech users through their own amendment of the scheme of phoneme. The other system of speech form or meaning form can be further subdivided into lexical scheme and syntactic scheme. The former is individualized and contains internal lexicons stored subconsciously. Besides, the context, which the lexicon occurs, also plays an important role in confirming the meaning. Except lexical scheme, the syntactic scheme also plays an equally important role in understanding the speech users. In order to understand the real meaning, it is a must for the reader or the listener to have a corresponding syntactic scheme, otherwise, the construction of meaning cannot be achieved.

The second level of scheme of language perception is situation structure, which also contains two systems. One is the knowledge system related to the speaker or the listener; the other system relates to the objects that are spoken. The first system refers to the relevant experiences or ideas that are brought by language perceivers' interpersonal relations or their present status. The second system refers to the perceivers experience speech users in a certain background. Under the situational structures, various ideas, experiences and embodiments are fully provoked and are cared and fulfilled by applying different systemic situational scheme from different angles and facets, so as to understand speech users' real meanings and speakers' real intensions (Wang, 2000).

The third level of scheme of language perception is intension structure. Each individual has the intension of being respected, accepted and admitted by others, and their intensions are deep-rooted and uncontrollable. There must be such kinds of intension structure in our scheme and let us unconsciously make different situational responses while facing different speech users. Generally speaking, the perception of speech is the process from form structure to situation structure and to intension structure.

\section{CONCLUSION}

Through the integration of the study of language perception in psycholinguistics during the past decades, we found that it was non-negligible to understand language perception macroscopically, and good language perception would be helpful for us to understand language. However, it does not suffice to study language perception macroscopically, because it cannot summarize language perception fully and precisely. If later scholars can associate the study of language perception with the microscopic aspects of speech recognition and written language perception, we sincerely believe that our study of language perception will forge ahead further. 


\section{REFERENCES}

[1] Guangqian Zhu. Literature and other [M]. Anhui: Education Press, 1996.

[2] Hailin Li. Theory of speech teaching [M]. Shanghai: Education Publishing House, 2000.

[3] Humboldt. On the differences in human language structure and its impact on the development of human spirit [M]. Beijing: commercial press, 1997.

[4] Jiping Pan. Psychological mechanism of language sense [J]. Journal of Hubei University, 1995.

[5] Sapir. Linguistic Theory [M]. Beijing: Commercial Press, 1985.

[6] Shangwen Wang. Language sense theory [M]. Shanghai: Shanghai Education Publishing House, 2000.

[7] Shengtao Ye. The Chinese education collection [M]. Education Science Press, 1980.

[8] Xinjun Lu. The history of the definition of language sense [J]. Modern Chinese, 2006.

[9] Xueyun $\mathrm{Hu}$. The concept of language sense and the law of language sense [J]. Foreign Language Teaching, 1992. 